\title{
Classificação das posições de terceiros molares inclusos e semi-inclusos: uma revisão de literatura
}

\author{
Classification of the positions of enclosed and semi-included third molars: a literature review
}

\author{
Amanda Botelho Galinari" *
}

Belo Horizonte, Minas Gerais, Brasil. *Autor para correspondência. E-mail: amandabggalinari@hotmail.com

\begin{abstract}
Resumo: Introdução: Os terceiros molares são os últimos dentes, que de acordo com escala cronológica de erupção dentária, tem o seu surgimento na arcada dentária e, habitualmente, se apresentam inclusos ou semi-inclusos. Revisão: Diversas podem ser as causas desse fenômeno, seja pela topografia óssea, pela falta de espaço na arcada ou ainda pela posição do segundo molar. Os dentes mais susceptíveis de serem encontrados inclusos são os terceiros molares superiores e inferiores e em seguida, os caninos superiores. Discussão: O dente incluso é aquele cuja erupção não ocorreu na época esperada segundo a cronologia eruptiva dos dentes permanentes, e diversas podem ser as causas e tipos de inclusão. Considerações finais: Pensando nisso, o presente artigo apresenta as principais classificações das posições dos terceiros molares retidos e a frequência em que estes podem ser mais comumente encontrados.
\end{abstract}

Palavras-chave: Classificação de Winter, Classificação de Pell e Gregory, terceiros molares inclusos, etiologia e prevalência de dentes inclusos.

\begin{abstract}
Introduction: Third molars are the last teeth that, according to the chronological scale of tooth eruption, emerge in the dental arch and are usually included or semi-included. Revision: There may be several causes for this phenomenon, either by bone topography, lack of space in the arch or by the position of the second molar. The teeth most likely to be found included are the upper and lower third molars and then the upper canines. Discussion: An impacted tooth is one whose eruption has not occurred at the expected time according to the eruption chronology of permanent teeth. Final Considerations: With this in mind, this article presents the main classifications of the positions of retained third molars and the frequency in which they are most commonly found.
\end{abstract}

Keywords: Winter's classification, Pell and Gregory's classification, included third molars, etiology and prevalence of included teeth.

\section{Introdução}

O dente incluso é descrito como sendo um dente cuja erupção não ocorreu na época prevista de acordo com a cronologia eruptiva dos dentes permanentes, e diversas podem ser as causas e tipos de inclusão. Frequentemente, os terceiros molares são os dentes que mais se encontram inclusos e são os que possuem maior demanda para a realização de extração. No entanto, a nomenclatura para esse fenômeno ainda exibe bastante divergência entre os pesquisadores da Odontologia atual. Assim, são diversas as terminologias, entre as quais, dente incluso, impactado, retido, não irrompido e dente serotino, são comumente observadas na literatura vigente.

As causas destes problemas ainda são desconhecidas, mas sabe-se que estas situações normalmente acontecem quando não há espaço suficiente no arco alveolar para o devido posicionamento do terceiro molar (Candeiro et al., 2009). Uma segunda possível justificativa para a inclusão de terceiros molares pode ser dada pelas modificações que podem ocorrer durante a formação do dente, pois durante o seu desenvolvimento, o terceiro molar muda continuamente a sua inclinação e se submete a rotações pré eruptivas solenes que ocorrem quando este se aproxima do segundo molar.

Dentre as consequências geradas da permanência de dentes inclusos e semi-inclusos, alguns autores citam que podem ocorrer reabsorções radiculares, doenças periodontais, formação de cistos e tumores odontogênicos, reabsorções externas do dente envolvido e lesões de cárie. Em contrapartida, há autores que defendem que em casos de terceiros molares inclusos assintomáticos não deve haver a remoção do elemento dentário, pois haveria mais chances de ocorrerem problemas advindos da cirurgia do que problemas causados pelo dente incluso em si, como, por exemplo, a parestesia, hemorragias, entre outros (Bishara, 1999). 
A radiografia panorâmica tem valiosa importância para o diagnóstico e planejamento cirúrgico ou ainda para a preservação do dente retido. Nesse sentido, as radiografias panorâmicas oferecem uma ampla visão sobre a condição do complexo maxilomandibular de todo o espaço dento-alveolar bem como as estruturas anatômicas circunvizinhas ao dente incluso. Se houver necessidade, o Cirurgião-Dentista pode utilizar de tomografias odontológicas e ressonância magnética para melhor diagnosticar o paciente.

Diante do exposto, o presente artigo de revisão integrativa da literatura tem o objetivo de identificar, e classificar os diferentes graus de impacção mais comumente observados nos terceiros molares inferiores e superiores em relação aos ossos do da mandíbula e da maxila, assim como a visão epidemiológica em três diferentes universidades brasileiras, fundamentando-se nas classificações dos tipos de inclusão propostas pelos pesquisadores Winter (1926) e Pell e Gregory (1933).

\section{Revisão}

O presente estudo se trata de uma revisão integrativa da literatura a qual possibilita a identificação de tendências ou evidências que fundamentam o estudo proposto. Foram utilizados os seguintes descritores: "Classificação de Winter"; " Classificação de Pell e Gregory”; "terceiros molares inclusos”; "etiologia e prevalência de dentes inclusos”. Estes termos foram verificados nos Descritores em Ciências da Saúde (Decs). A busca de publicações ocorreu no período de agosto de 2021 no portal da BVS e SciELO, não foi considerado o período de tempo.

Foram incluídos os artigos que abordavam a disfunção a classificação de terceiros molares inclusos, suas complicações, prevalência e possíveis tratamentos. Os filtros utilizados foram: artigos disponíveis no idioma Português que envolviam a classificação de terceiros molares inclusos, classificação de Winter e a classificação de Pell e Gregory. Sendo que foram acrescentados os filtros: LILACS e BBO. O critério de seleção dos artigos científicos foi a leitura do resumo.

Muitos pacientes que possuem ao menos um elemento dentário retido desconhecem essa situação, mas sabe-se que aproximadamente $65 \%$ da população mundial jovem possui um dente retido (Castro Neto, 2009), sendo que só tomam conhecimento dessa situação após sentirem dores na região do dente acometido. De acordo com Graziani (1995) os terceiros molares inferiores são os dentes mais acometidos, em seguida são os caninos superiores, caninos inferiores, pré-molares superiores, pré-molares inferiores, incisivos, e por fim, os primeiros e segundos molares. Para o diagnóstico, é necessário que o cirurgião-dentista utilize de exames radiográficos complementares, sendo que, usualmente a radiografia panorâmica é a mais indicada.

O elemento dentário é classificado como incluso e semi-incluso de acordo com o grau de retenção e de acordo com a natureza do tecido que o recobre. O dente totalmente retido pode se apresentar de duas maneiras diferentes: a primeira é quando o dente não rompe a cortical óssea e se apresenta coberto por osso e por mucosa. E a segunda maneira é quando o dente consegue romper a cortical óssea, mas não consegue ultrapassar a barreira submucosa (gengiva). E classifica-se como semi-incluso o dente que se apresenta parcialmente erupcionado em boca, ou seja, o dente consegue ultrapassar a cortical óssea e a submucosa de forma incompleta.

Dentre os principais fatores que podem levar à impactação dentária, a falta de espaço na arcada dentária e o crescimento limitado da mandíbula e/ou maxila são considerados importantes fatores etiológicos que resultam nesse fenômeno da impactação dentária (De Tobel et al., 2017). Muitas vezes, o dente incluso é assintomático mas isso não impossibilita que surjam patologias como por exemplo, cisto dentígero, cárie, perda óssea, pericoronarite, reabsorção da raiz do dente adjacente, entre outros. Em consequência, os sintomas normalmente incluem dor na região acometida, edema na gengiva e na face, abscesso, trismo e halitose (Yilmaz et al., 2016).

Ainda hoje há bastante discussão acerca da conduta clínica diante dessa situação, alguns autores defendem que deve-se realizar a extração preventiva, outros em contrapartida, defendem que não há necessidade para exodontia de um dente incluso assintomático. Caso o profissional opte pela extração, a posição anatômica dos dentes inclusos consistem em um importante fator que deve ser levado em consideração durante o planejamento cirúrgico.

Existem diferentes classificações para o grau de inclusão dos terceiros molares inferiores, de acordo com a classificação de Winter (1926), o terceiro molar retido pode ser classificado de acordo com o seu posicionamento em relação ao segundo molar adjacente. Já a classificação proposta por PELL \& GREGORY (1933), sugere a categorização do terceiro molar inferior retido em relação ao plano oclusal e entre a distal do segundo molar e o ramo da mandíbula. 
Proposta em 1926, a classificação de Winter foi uma das primeiras tentativas para categorizar as posições dos terceiros molares inclusos. Para isso, ele levou em consideração a inclinação do longo eixo do terceiro molar impactado em relação ao segundo molar adjacente (Tabela 1).

Tabela 1. Classificação de Winter.

\begin{tabular}{|c|c|}
\hline Posição do dente incluso & Definição \\
\hline Mesioangular & O dente incluso está direcionado para a face mesial \\
\hline Distoangular & O dente incluso está direcionado para a face distal em relação ao longo eixo do segundo molar \\
\hline Vertical & O dente incluso se encontra paralelo ao longo eixo do segundo molar \\
\hline Horizontal & $\begin{array}{l}\text { O dente incluso está em uma angulação tão acentuada que se encontra perpendicular ao longo } \\
\text { eixo do segundo molar }\end{array}$ \\
\hline Invertido & $\begin{array}{c}\text { O dente incluso está com a face oclusal voltada para a base da mandíbula e, dessa forma, se } \\
\text { encontra de "cabeça para baixo" }\end{array}$ \\
\hline Vestibuloversão e linguoversão & $\begin{array}{c}\text { O terceiro molar incluso se encontra totalmente horizontalizado, isto é, inclinado para } \\
\text { vestibular ou lingual }\end{array}$ \\
\hline
\end{tabular}

Posteriormente, em 1933, Pell \& Gregory, compararam a parte elevada da face oclusal do terceiro molar inferior retido em relação à porção cervical do segundo molar adjacente, tal comparação serviu como referência para que esses pesquisadores produzissem um sistema de classificação que dividia os resultados em três classes: A, B ou C tomando como base a profundidade desse elemento retido no osso da mandíbula (Tabela 2).

Tabela 2. Posição do dente em relação ao plano oclusal.

\begin{tabular}{|c|c|}
\hline $\begin{array}{l}\text { Posição do dente em relação } \\
\text { ao plano oclusal }\end{array}$ & Definição \\
\hline Posição A & $\begin{array}{l}\text { Nota-se que a parte mais alta do terceiro molar inferior está na mesma altura ou um pouco } \\
\text { acima do segundo molar. }\end{array}$ \\
\hline Posição B & $\begin{array}{c}\text { Nota-se que a parte mais alta da face oclusal do terceiro molar inferior está abaixo da altura do } \\
\text { segundo molar. }\end{array}$ \\
\hline Posição C & $\begin{array}{l}\text { Nota-se que a parte mais alta da face oclusal do terceiro molar inferior está na mesma altura ou } \\
\text { um pouco abaixo da região cervical do segundo molar. }\end{array}$ \\
\hline
\end{tabular}

Outra classificação fora proposta por esses mesmos autores e dessa vez, relacionava a amplitude do sentido mésio-distal do terceiro molar inferior incluso em relação ao ramo mandibular, sendo subdivididos em classes, a saber, classe I, II ou III (Tabela 3).

Tabela 3. Classificação de Pell e Gregory.

\begin{tabular}{lc}
\hline $\begin{array}{l}\text { Classificação de } \\
\text { Pell \& Gregory }\end{array}$ & Definição \\
\hline Classe I & $\begin{array}{c}\text { Verifica-se que há espaço hábil entre a margem anterior da mandíbula e a face distal do segundo molar } \\
\text { inferior, para alocar a coroa do terceiro molar inferior. }\end{array}$ \\
\hline Classe II & $\begin{array}{c}\text { Verifica-se que o espaço entre a margem anterior do ramo mandibular e a face distal do segundo molar } \\
\text { inferior é menor que o espaço necessário para que a coroa do terceiro molar inferior seja alocada. }\end{array}$ \\
\hline Classe III & Verifica-se que há indisponibilidade de espaço para que o terceiro molar inferior seja alocado no arco \\
alveolar.
\end{tabular}


Para além disso, o terceiro molar incluso pode ser classificado de acordo com o grau de impactação: intraósseo, semi-incluso e submucoso. Assim, o dente é tido como incluso intraósseo quando ele não erupciona na cavidade oral e está completamente rodeado por osso e mucosa. Considera-se como semiincluso o dente que se apresenta parcialmente retido em osso e/ou mucosa, isto é, o dente se apresenta parcialmente erupcionado na cavidade bucal. E por fim, um dente pode ser retido somente pela submucosa, ou seja, o dente consegue perfurar a cortical óssea mas continua coberto por gengiva.

No caso dos dentes superiores, eles podem ser classificados seguindo os mesmos critérios dos dentes inferiores, com exceção do sistema proposto por Winter. Assim, os dentes superiores seguem a classificação segundo a natureza do tecido que o recobre; de acordo com o posicionamento do terceiro molar no sentido ocluso-apical e entre a distal do elemento incluso e o ramo da mandíbula.

\section{Discussão}

Uma pesquisa feita na clínica escola de Odontologia da Universidade Estadual de Feira de Santana (UEFS), apontou que através da análise de 88 prontuários de pacientes com idades entre 24 e 51 anos que foram submetidos a extração de dentes inclusos na disciplina de Cirurgia da UEFS, no período de janeiro de 2001 a janeiro de 2002, os resultados obtidos mostraram que a faixa etária de 20 a 29 anos possuem maior disposição para a prevalência de dentes inclusos (73,9\%), sendo que a distribuição por gênero demonstrou que $59,1 \%$ da amostra eram mulheres e 40,9\%, homens. Através dessa pesquisa os autores também concluíram de acordo com a classificação de Pell e Gregory (1933) e de Winter (1926) o dente incluso de maior prevalência foi o terceiro molar inferior $(72,8 \%)$ sendo que a posição de inclusão mais comum para esse elemento foi a vertical $(36,9 \%)$ e em seguida, a posição mesioangular (32,0\%), além disso, a posição A foi a mais comum entre os pacientes $(47,6 \%)$ e em seguida a classe B foi a mais observada (43,7\%). Em relação aos dentes superiores, a posição de inclusão mais comum para esse elemento foi a posição vertical $(49,3 \%)$ e em segundo lugar a posição a distoangular (39,0\%), em relação ao plano oclusal, a classe B e C foram mais prevalentes com 36,35\% cada uma, ou seja, apresentaram valores iguais (Farias et al., 2003).

Também foi realizada uma pesquisa quanto a prevalência dos tipos de impacção dos terceiros molares na clínica escola do Centro Universitário de Anápolis - UniEVANGÉLICA no ano de 2019, para essa pesquisa foram avaliados 1041 pacientes, sendo que o terceiro molar inferior esquerdo foi encontrado com grande predominância na posição de incluso (524 pacientes), a posição A foi encontrada em $65 \%$ dos casos enquanto que o terceiro molar inferior direito foi encontrado em menor prevalência (517 pacientes), com prevalência da posição A em $68 \%$ dos casos. Sobre os molares superiores, notou-se a prevalência da posição A, sendo $59 \%$ do lado direito e $58 \%$ do lado esquerdo. Contudo, a análise feita seguindo a classificação de Winter (1926), infere que 2129 elementos foram classificados, e os achados mostram que os terceiros molares superiores possuem predominância na posição vertical, ambos com $81 \%$ seguida da posição distoangular. Para os terceiros molares inferiores, notou-se a predominância da posição de $62 \%$ e $64 \%$ para o lado esquerdo e direito, respectivamente. Em seguida, a posição mesioangular foi a mais observada, sendo $23 \%$ do lado esquerdo e $22 \%$ dos casos no lado direito (Lopes et al., 2019).

Ademais, foram examinadas 297 radiografias panorâmicas de pacientes que foram atendidos entre o período de março de 2007 a março de 2008 na na Clínica de Radiologia da Universidade Federal do Ceará, sendo que 122 pertenciam ao sexo masculino e 175 ao sexo feminino, com idades que variavam entre 15 a 60 anos. A partir dos resultados obtidos, os pesquisadores concluíram que, segundo a classificação de Winter (1926), os terceiros molares superiores apresentaram mais prevalência na posição vertical (64,4\%) e para os terceiros molares inferiores, a prevalência foi da posição mesioangular $(52,5 \%)$. Já de acordo com a classificação de Pell e Gregory (1936), os terceiros molares demonstraram a predominância da posição A $(59,0 \%)$ e em seguida a posição C (28,5\%). Nos dentes inferiores, a prevalência foi da posição A (59,7\%) seguida da posição $B(25,0 \%)$. Além do que, a classe I foi a mais observada em relação à posição do terceiro molar inferior e o ramo da mandíbula $(63,0 \%)$, em seguida a classe II $(32,5 \%)$ foi a mais observada no estudo (Candeiro et al., 2009).

A remoção cirúrgica dos terceiros molares requer um planejamento minucioso e bastante destreza desde o diagnóstico até a exodontia, várias técnicas podem ser utilizadas nesse momento de acordo com a classificação da posição e inclinação do terceiro molar a ser extraído. Portanto, nota-se que as classificações propostas por Winter e Pell \& Gregory são fundamentais para que o cirurgião-dentista conheça a anatomia e prováveis intercorrências durante o ato cirúrgico. Assim, a partir dos estudos de Winter (1926), pode-se observar que é nítida a prevalência de inclusão dos terceiros molares mandibulares na posição mesioangular. Em relação aos terceiros molares maxilares, observa-se a predominância da posição vertical. Sobre a posição 
do terceiro molar inferior em relação ao plano oclusal do segundo molar (Pell \& Gregory, 1936), pode-se verificar que nos terceiros molares inferiores e superiores há prevalência da posição A, além disso, pode-se notar que há prevalência da classe I.

\section{Considerações finais}

A cirurgia para extração dos terceiros molares inclusos é um procedimento habitual para o Cirurgião Bucomaxilofacial, e para o Cirurgião-Dentista Clínico Geral; no entanto, antes de decidir extrair ou não os terceiros molares inclusos e semi-inclusos o Cirurgião-Dentista deve se atentar para as características e posições que os dentes se encontram, para que de forma cuidadosa, realize a extração, pois tal procedimento está diretamente relacionado com uma série de acidentes e complicações.

\section{Referências}

Bishara, S. E. 1999. Third molars: a dilemma! Or is it?. American Journal of Orthodontics and Dentofacial Orthopedics, 115(6), 628-633.

Candeiro, G. T. D. M., Correia, F. C., \& Candeiro, S. A. L. D. M. 2009. Ulectomia como opção cirúrgica no retardo da erupção dentária: relato de caso. Revista de Odontologia, 45-49.

Castro Neto, F. M. P. 2009. Avaliação da indicação de extração dos terceiros molares numa população portuguesa. Dissertação de mestrado. Porto, PT: Universidade do Porto.

De Tobel, J., Radesh, P., Vandermeulen, D., \& Thevissen, P. W. 2017. An automated technique to stage lower third molar development on panoramic radiographs for age estimation: a pilot study. The Journal of forensic odonto-stomatology, 35(2), 42.

Farias, J. G. D., Santos, F. A. P. D., Campos, P. S. F., Sarmento, V. D. A., Barreto, S., \& Rios, V. 2003. Prevalência de dentes inclusos em pacientes atendidos na disciplina de cirurgia do curso de Odontologia da Universidade Estadual de Feira de Santana. Pesquisa Brasileira em Odontopediatria e Clínica Integrada, 3(2), 15-9.

Graziani, M. 1995. Cirurgia buco-maxilo-facial (7a ed.). Rio de Janeiro, RJ: Guanabara Koogan.

Lopes, L. S., da Silva Morais, M. N., Ferreira, M. U., de Paula, L. G. F., \& Mariano-JÚnior, W. J. 2019. Prevalência dos tipos de impacção de terceiros molares na clínica odontológica de ensino do centro universitário de Anápolis-UniEVANGÉLICA. Sci Invest Dent,24(1), 13-22.

Pell, G. J., \& Gregory, G. T. 1933. Impacted mandibular third molars: classifications and modified technique for removal. Dent Digest, 39, 330.

Winter, G. B. 1926. Impacted mandibular third molars. St. Louis: Med Book Co.

Yilmaz, S., Adisen, M. Z., Misirlioglu, M., \& Yorubulut, S. 2016. Assessment of third molar impaction pattern and associated clinical symptoms in a central anatolian turkish population. Medical Principles and Practice, 25(2), 169-175.

\section{Minicurrículo}

Amanda Botelho Galinari. Graduanda no curso de Odontologia na Faculdade Pitágoras de Belo Horizonte, Belo Horizonte, Minas Gerais (MG), Brasil.

Como citar: Galinari, A.B. 2021. Classificação das posições de terceiros molares inclusos e semi-inclusos: uma revisão de literatura. Pubsaúde, 7, a239. DOI: https://dx.doi.org/10.31533/pubsaude7.a239

Recebido: 10 ago. 2021.

Revisado e aceito: 24 ago. 2021.

Conflito de interesse: os autores declaram, em relação aos produtos e companhias descritos nesse artigo, não ter interesses associativos, comerciais, de propriedade ou financeiros que representem conflito de interesse.

Licenciamento: Este artigo é publicado na modalidade Acesso Aberto sob a licença Creative Commons Atribuição 4.0 (CC-BY 4.0). 